\title{
Liquid Chromatography with Tandem Mass Spectrometry-Based Proteomic Discovery in Aging and Alzheimer's Disease
}

\author{
Thomas J. Montine, Randall L. Woltjer, Catherine Pan, Kathleen S. Montine, and Jing Zhang \\ Department of Pathology, University of Washington, Harborview Medical Center, Seattle, Washington 98104
}

\begin{abstract}
Summary: Systems biology offers enormous potential to understand the complexity of human brain aging and neurodegenerative diseases. Proteomics has an important role in these investigations because of its unique strengths and because of the potential central pathogenic contribution of pathological protein to several of these diseases. Here we have reviewed the methods and presented some examples of liquid chromatography-electrospray ionization-tandem mass spectrometry-
\end{abstract}

based proteomics, with and without quantification using isotope-coded affinity tags, in the investigation of aging and Alzheimer's disease. As protocols and methods for improved quantitative high-throughput proteomics constantly improve, this approach will likely continue to provide deeper insight into human brain aging and neurodegenerative diseases. Key Words: Proteomics, Alzheimer's disease, cerebrospinal fluid (CSF), neurofibrillary tangles.

\section{DISCOVERY SCIENCE IN NEURODEGENERATIVE DISEASES}

Recent technical innovations have yielded new opportunities to simultaneously investigate large numbers of gene transcripts and proteins that just a few years ago would not have been possible, spawning a new area of biomedical research called discovery science. ${ }^{1}$ Genomics, the unbiased simultaneous assessment of relative changes in thousands of gene transcripts, is now a widely employed approach to assess relevance of molecular pathways using a variety of platforms that are becoming standardized; for examples, see the reviews by Ginsberg and coworkers, ${ }^{2}$ as well as Miller and Federoff ${ }^{3}$ in this issue. Proteomics, also an unbiased method that simultaneously assesses thousands of proteins, is less standardized than genomics, but likely will have an equally large influence on our understanding of molecular physiology and pathophysiology. In this article we will focus on a type of proteomic analysis; however, the reader is also directed to other proteomic articles in this issue. ${ }^{4,5}$

The magnitude of the human genome and proteome is worthy of our consideration. In humans, the entire complement of protein-coding genes is now fairly solidly

Address correspondence and reprint requests to: Thomas Montine, M.D., Ph.D., University of Washington, Harborview Medical Center, Department of Pathology, Box 359791, Seattle, WA 98104. E-mail: tmontine@u.washington.edu estimated at between 20,000 and 25,000 , a surprisingly low number. There are a larger number of transcripts from the human genome that are not apparently translated, an obvious issue to be reconciled in gene transcription studies. Estimates on the total number of proteins vary widely from about 400,000 to several million, a reflection of our ignorance of post-translational modifications and alternative splicing of transcripts. Regardless, there are many-fold more proteins than genes. The situation with gene transcripts and protein is further complicated because, unlike the genome that is relatively constant from cell to cell within an organism, transcription profiles and protein composition vary widely among cell types and tissues, and protein composition varies widely among body fluids. From these considerations derives the concept of "one genome but many proteomes." This is fundamentally important when evaluating data from discovery science approaches. While it is entirely appropriate to evaluate the genome of blood lymphocytes for brain disease-associated mutations, it is not at all clear what relationships exist between either the lymphocyte transcriptome or plasma proteome and the hippocampal pyramidal neuron transcriptome or proteome, for example. The same problem plagues the proteome of different body fluids such as plasma and cerebrospinal fluid (CSF).

There are several reasons why we think that proteomics is more tractable than gene expression analyses of 
human neurodegenerative diseases. First is the general point that proteins, not gene transcripts, are the main functional endpoints of gene expression, and it is well established that there is often a poor correlation between mRNA levels and the level of translated proteins. Second, a specific limitation of transcriptomics in human neurodegenerative disease is that-unlike many other diseases-gene transcripts must be studied in tissue that is almost exclusively obtained postmortem. Stability of gene transcripts in postmortem tissue is problematic; while some rapidly obtained tissues show acceptable overall mRNA fidelity, the stability of individual transcripts is highly variable and even with optimally obtained tissue some unknown subset of transcripts will be lost. $^{6}$ Unlike gene transcripts, translated proteins are much more stable, thereby largely removing concerns over significant differences in stability during a short postmortem interval. Third, a dominant model of several common and uncommon neurodegenerative diseases is that these are diseases of protein misfolding, a process that would be transparent to transcription analysis. Finally, while mRNA cannot be assessed intra vitam in $\mathrm{CSF}$, it is well established that brain-derived proteins, tau and phosphorylated (P)-taus for example, can be quantified in CSF from the lumbar cistern and used as biomarkers of neurologic disease. There are well-known limitations that accompany investigating CSF as a reflection of brain metabolism; however, the tremendous advantage of CSF is that it permits investigation of patients early in the course of disease, at the time when new therapies are needed.

While discovery methods are truly impressive in their capacity to investigate thousands of products simultaneously in an unbiased manner, they are limited in that they do not specify mechanism. Indeed, as experience is gained in these techniques, it is becoming clear that they should be considered highly sophisticated, unbiased screening tools that establish relevance but not mechanistic connections. Moreover, these highly sophisticated screens require careful design, evaluation, confirmation in the same samples by alternative methods, and validation in different sample sets.

\section{A QUANTITATIVE PROTEOMIC METHOD USING LIQUID CHROMATOGRAPHY-TANDEM MASS SPECTROMETRY}

The initial step in any proteomic analysis is the establishment of the "analytical proteome" under investigation. Here, basic but critically important biochemical issues come into play as protein fractions are isolated or enriched. Protein separation or isolation is typically achieved by one of three approaches: "protein chips" or activated surfaces that bind proteins based on chemical characteristics or two-dimensional (2-D) gel electrophoresis $;{ }^{7,8}$ such methods are used in other articles in this issue including Butterfield and coworkers ${ }^{4}$ and Choe and coworkers. ${ }^{9}$ In contrast to these approaches, liquid chromatography (LC) also can be used to separate peptides before direct introduction to tandem mass spectrometry (MS). Indeed, proteomic analysis using LC-MS-MS involves four fundamental steps: (i) peptide separation or isolation, (ii) ionization, (iii) mass analysis, and (iv) database searching to infer protein identity.

Liquid chromatography has tremendous capacity and versatility in resolving power especially when multiple solid phase columns are combined, approximately 100fold greater sensitivity than other methods to low abundance proteins, and easy coupling to MS. ${ }^{9-11}$ For the second step, peptide ionization, the two major ionization techniques are matrix assisted laser desorption/ionization (MALDI) and electrospray ionization (ESI), with LC typically coupled to ESI in the past. However, with the introduction of seamless integration of LC and matrix spotting systems, it is now also possible to combine peptide separation by LC with MALDI. ${ }^{12}$ Step three, mass analysis, can be achieved in time with ion trap mass spectrometers that are commonly coupled to LC-ESI systems, or in space with time-of-flight (TOF) mass spectrometers that are commonly coupled to MALDI systems. Thus it is now possible to combine the power of peptide separation by LC with the high resolution of MALDI-TOF-TOF instruments. Other types of mass analyzers include "hybrid" mass spectrometers such as the quadrupole-TOF (QqTOF) ${ }^{13}$ and the linear ion-trapFourier transform ion cyclotron resonance (LTQ-FT). ${ }^{14}$ The last step whereby tandem mass spectral data are used to infer protein identity by database searching is arduous, complex, and still evolving. Recent consensus criteria are a move in the right direction and undoubtedly will be an area of increased focus in the future.

It is very important to stress the point of quantification. In transcriptomics, not only is the transcript identified, but the relative quantity of that transcript in two conditions is determined. Largely, because of the variation in peptide ionization, while all proteomic methods can identify peptides and infer protein identity, none are quantitative. One way to circumvent this shortcoming is to use proteomic approaches solely to identify candidates and then validate and quantify with alternative techniques; we will show examples of this approach below. Of course, a limitation to this approach is that validation techniques tend to have much lower throughput and so this approach negates one of the major strengths of proteomics. Ideally, one wants to incorporate quantitation into the proteomic approach being used. In LC-based proteomics, quantification, especially in high throughput mode, is achieved by incorporation of stable isotopes on the peptides being analyzed. One widely employed tech- 
nique for stable isotope incorporation is Isotope Coded Affinity Tagging (ICAT). ${ }^{10}$

The conventional ICAT probe consists of a biotin tag, a linker, and an iodoacetamide handle. The linker contains either a light $\left({ }^{12} \mathrm{C}\right)$ or a heavy $\left({ }^{13} \mathrm{C}\right)$ component, forming two probes with indistinguishable chemical behavior but with a mass difference of $9 \mathrm{Da}$. The concept behind ICAT that overcomes the variable nature of MS ionization is conceptually similar to gene microarrays that quantify relative abundance by mixing control and experimental samples separately labeled with different fluorescent dyes and determining the ratio of fluorescence at the two wavelengths. ICAT mixes control and experimental samples labeled with heavy or light "tags" that can be retrieved with an avidin affinity column, separated by LC, and resolved by ESI-MS. While ionization of the molecules in the MS remains variable, the light and heavy tagged peptides are being ionized simultaneously, thereby allowing protein identification and quantification of relative abundance. The creators of the ICAT method also have developed an algorithm for the automated statistical analysis of protein abundance ratios (ASAPRatio) to interpret data from ICAT tagged peptides; ${ }^{15}$ an advantage of this over 2-D gels is that ASAPRatio utilizes relative quantitative data from all peptides belonging to a protein rather than a single peptide. Of course, ICAT technique is not without its limitations, the major one being that ICAT labeling is limited to cysteinyl-containing peptides and comparison of only two groups. To circumvent these limitations, new techniques have been developed, including amine-reactive isobaric tagging reagents (ITRAQ), which not only allows quantification of all peptides but also direct comparison of up to four samples simultaneously. ${ }^{16}$

\section{EXAMPLES OF LC-MS-MS-BASED PROTEOMICS OF AGING AND ALZHEIMER'S DISEASE}

Many research groups are now testing hypotheses about neurodegenerative diseases using proteomic techniques and several other articles in this volume reflect this activity. Here we will focus on two examples from our laboratories that highlight the utility of LC-MS-MS techniques to proteomics of aging and Alzheimer's disease $(A D)$.

\section{Example 1: laser capture microdissected neurofibrillary tangles}

A feature of many neurodegenerative diseases is the accumulation of abnormal proteins in hallmark pathologic inclusions; in AD these are predominantly amyloid- $\beta(\mathrm{A} \beta)$ in senile plaques (SPs) and tau in neurofibrillary tangles (NFTs). ${ }^{17}$ SPs in AD are complex structures that contain not only fibrillar aggregates of
A $\beta$ peptides but also reactive glial and varyingly damaged neuronal processes. Inheritance of mutations in the gene encoding the precursor protein of $\mathrm{A} \beta$ peptides as well as genes encoding proteins that alter amyloid precursor protein (APP) metabolism are associated with rare autosomal dominant forms of $\mathrm{AD} .^{18}$ NFTs are the other hallmark histopathologic feature of AD. A hyperphosphorylated abnormal form of the microtubule binding protein tau, paired helical filament (PHF)-tau, has been identified as the major protein component of NFTs. ${ }^{19}$ Inheritance of mutations in the gene for tau is associated with a related form of dementia that shares with AD NFT but not SP formation. $^{20}$

Limited analysis of proteins associated with NFTs has given important new clues to the pathogenesis of neurodegenerative diseases. For example, apolipoprotein (apo) E was colocalized to NFTs and SPs before genetic association studies that linked inheritance of the $\varepsilon 4$ allele of $A P O E$ with increased risk of late-onset familial and sporadic AD. ${ }^{21}$ Similarly, $\alpha$-synuclein was identified as the major nonamyloid component of SPs using traditional techniques before discovering that this protein is central to a number of neurodegenerative diseases now called $\alpha$-synucleinopathies. ${ }^{22}$ Others have reported proteomic studies of SPs. ${ }^{23}$ We undertook a proteomic analysis of laser-captured microdissected (LCM) NFTs. ${ }^{24}$

NFTs were visualized by anti-tau immunohistochemistry and obtained by LCM from pyramidal neurons in hippocampal sector CA1, solubilized in formic acid with sonication, digested with trypsin, the resulting peptides analyzed by LC-MS-MS, and the data analyzed by SEQUEST against the International Protein Index database followed by probability calculation with PeptideProphet and ProteinProphet. We identified a total of 155 proteins in laser captured NFTs, 72 of which were identified by multiple unique peptides. ${ }^{24}$ Of the 72,63 proteins had no previously known association with NFTs (Table 1). Expected proteins, such as tau and apo E, were identified in our proteomic screen, confirming the ability of this approach to identify NFT-associated proteins. We validated by immunohistochemistry that glyceraldehyde-3-phosphate dehydrogenase (GAPDH), one of the proteins identified in our investigation that had not been previously associated with NFTs, colocalized with the majority of NFTs as well as plaque-like structures in AD brain and was coimmunoprecipitated by antibodies to PHFtau, but not tau, in temporal cortex. ${ }^{24}$ We further characterized the pathological association of GAPDH with $\mathrm{AD}$ by showing that it, along with P-tau and $\mathrm{A} \beta$ peptides, was present in detergent-insoluble fractions from AD temporal cortex but not from age-matched controls. These data are the first proteomic investigation of NFTs. Moreover, our results validate this approach by demonstrating that GAPDH, a glycolytic and microtubule bind- 
TABLE 1. Proteins Identified by LC-ESI-MS-MS in LCM NFTs from Hippocampal Sector CAI

Cytoskeletal or Structural

Vimentin*

Cofilin

Neurofilament $68 \mathrm{kDa}$

Myelin basic protein, splice isoforms 1, 3, 4, 5, and 6

Microtubule-associated protein tau isoforms $1,2,3$, and $4 *$

Tubulin alpha and beta chains*

Actin, cytoplasmic 1 and $2 *$

Septin 7

Alpha-internexin

Energy and Metabolism

Adolase C, fructose-biphosphate

Malate dehydrogenase, mitochondrial precursor

Glucose-6-phosphate isomerase

Hexokinase, multiple splice isoforms

ATP synthase alpha and beta chains, mitochondrial precursor

Fructose-bisphosphate aldolase A

Creatine kinase, B chain

Sodium/potassium-transporting ATPase beta-1 chain, multiple splice isoforms

ADP, ATP carrier protein, multiple isoforms

Pyruvate kinase isozymes

Ferritin light chain

Lactate dehydrogenase $\mathrm{B}$

Cytochrome $c$ oxidase subunit IV isoform 1, mitochondrial precursor

Glyceraldehyde-3-phosphate dehydrogenase

Cytosolic malate dehydrogenase

Phosphoglycerate kinase 1

ATP synthase oligomycin sensitivity conferral protein, mitochondrial precursor

Carbonic anhydrase II

Elongation factor 1-alpha 1

Alpha enolase

Extracellular Matrix or Cell Adhesion

Brain link protein-1

Versican core protein precursor, multiple splice isoforms

Spectrin alpha and beta chains, brain

Tenascin-R

Desmoplakin

Desmoglein 1

Galectin 7

Intracellular Trafficking or Synaptic

Myc box dependent interacting protein 1, multiple isoforms

Vacuolar ATP synthase catalytic subunit A, ubiquitous isoform

Clathrin heavy chain 1

SH3-containing GRB2-like protein 2

Syntaxin binding protein 1 , splice isoforms 1 and 2

Dynamin-1

Synapsin IA, IIA, and IB

Apha-synuclein, isoforms 1, 2, and 4

Nuclear

$\mathrm{H} 1, \mathrm{H} 2 \mathrm{~A}, \mathrm{H} 4$ histone, multiple members

Heterogeneous nuclear ribonucleoprotein $\mathrm{K}$, isoforms 1 and 2

Signaling

Guanine nucleotide binding protein alpha

2',3'-cyclic nucleotide $3^{\prime}$-phosphodiesterase, multiple isoforms

Annexin A5

Guanine nucleotide-binding protein, $\mathrm{G}_{\mathrm{I}} / \mathrm{G}_{\mathrm{S}} / \mathrm{G}_{\mathrm{T}}$ beta subunits 1,2 , and 4

Microtubule-associated protein RP/EB family member 3

Mitogen-activated protein kinase $1 *$

Calcium/calmodulin-dependent protein kinase type II alpha chain* 
TABLE 1. Continued

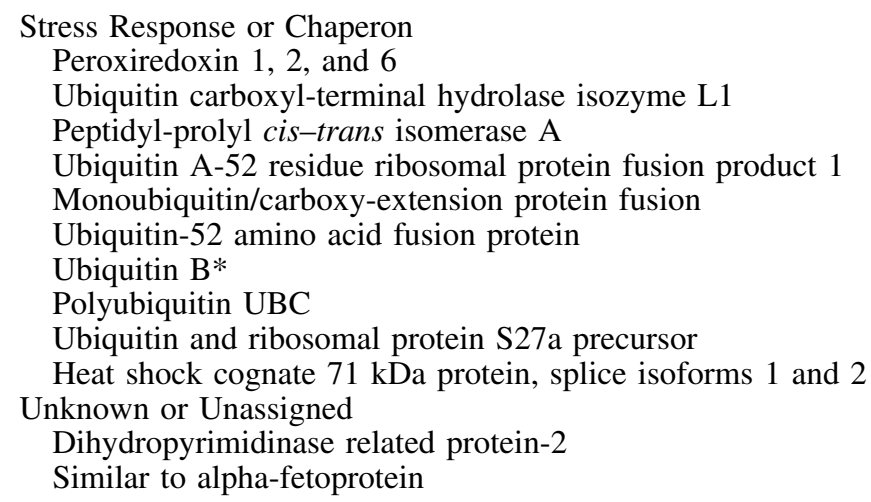

Hypothetical proteins were dropped from table. *Proteins already known to be associated with NFTs.

ing protein, not only colocalized to NFTs and immunoprecipitated with PHF-tau, but also is one of the few proteins known to undergo conversion to a detergentinsoluble form in AD.

GAPDH, unequivocally associated with NFTs and PHF-tau in our study, was initially described as a glycolytic enzyme that is now appreciated to have pleotrophic actions independent of energy metabolism that may influence neurodegeneration; these include roles in membrane fusion and endocytosis, microtubule binding, regulation of mRNA, and nuclear translocation in apoptosis. ${ }^{25}$ Others have immunohistochemically colocalized GAPDH to Lewy bodies in Parkinson's disease and proteins containing trinucleotide repeat expansions, such as huntingtin. ${ }^{26}$ With respect to AD, GAPDH has been shown to bind amyloid precursor protein in vitro, and cultured human fibroblasts from AD patients have abnormal subcellular distribution of GAPDH. ${ }^{27} \mathrm{Al}-$ though we discovered many NFT-associated proteins that are yet to be evaluated, our results with GAPDH highlight the power of unbiased proteomic techniques in pursuit of interacting proteins that may influence the pathogenesis of neurodegenerative diseases and show that GAPDH should be included with those few proteins that accumulate in inclusions and shift to a detergentinsoluble form in AD. Indeed, others reported simultaneously with our proteomic work an association between risk for $\mathrm{AD}$ and polymorphisms in the gene for GAPDH. ${ }^{28}$

\section{Example 2: human cerebrospinal fluid proteome}

While discovery of genes and pathological proteins is key to understanding the molecular pathogenesis of $\mathrm{AD}$, there also are pressing clinical needs. One of these is a reliable panel of surrogates or biomarkers that can be used to efficiently and reliably guide diagnosis and monitoring of response treatment. While many groups are actively investigating biomarkers, unfortunately single molecules or small groups of molecules have not yet significantly altered the clinical management of patients with AD. Moreover, although peripheral sampling of biomarkers, such as in plasma or urine, is highly desirable compared with CSF, as yet peripheral biomarkers have not been reproducibly applied to any of these diseases. ${ }^{29-32}$ However, some progress continues to be made in CSF biomarkers of AD using quantification of $\mathrm{A} \beta$ peptides, $\mathrm{P}$-taus, and markers of oxidative damage, especially $\mathrm{F}_{2}$-isoprostanes ( $\mathrm{F}_{2}$-IsoPs) and $\mathrm{F}_{4}$-Neuroprostanes. ${ }^{31,33-35}$ A theoretical limitation to these approaches is that they presuppose that molecules involved in the known pathogenic pathways of disease will also be the most discriminating biomarkers and, although this is a useful initial assumption, it need not be true. A second, practical limitation with existing biomarkers of $\mathrm{AD}$ is that, although control individuals typically show normally distributed values, patient values have a much broader distribution, suggesting significant heterogeneity among patients. This heterogeneity may be a reflection of inherently different subsets within what is currently called late-onset $\mathrm{AD}$. One approach to addressing both of these limitations would be to conduct a large-scale unbiased survey of potential CSF biomarkers to define an ensemble of biomarkers that discriminate among the common substrates of geriatric dementia and possibly among subsets within different dementing illnesses.

To begin to address these problems, we have undertaken high-throughput proteomic analysis of human CSF obtained from the lumbar cistern using LC-ESI-MS-MS with ICAT labeling. In our initial study, we used progressive organic extractions of pooled CSF to enrich for low abundance proteins from the younger $(n=22$, mean age $=25$ years $)$ and older $(n=16$, mean age $=72$ years $)$ individuals carefully demonstrated to be free of neurologic or psychiatric disease or medications that target the nervous system. ${ }^{36}$ We identified 315 proteins in human CSF. The average ratio was calculated with all of the peptides detected for each individual protein using the 
TABLE 2. CSF Proteins in Older versus Younger Individuals

\begin{tabular}{|c|c|c|}
\hline & $\geq 100 \%$ & $\geq 50 \%$ but $<100 \%$ \\
\hline Decreased in older & Agrin & $\begin{array}{l}\text { 1. Haptoglobin } \\
\text { 2. Nuclear autoantigenic sperm } \\
\text { protein isoforms }\end{array}$ \\
\hline Increased in older & $\begin{array}{l}\text { 1. Bromodomain adjacent to zinc } \\
\text { finger domain protein } 1 \mathrm{~B} \\
\text { 2. Serine/threonine protein } \\
\text { phosphatase PPI subunits } \\
\text { 3. Ubiquinol-cytochrome } c \\
\text { reductase core protein I }\end{array}$ & $\begin{array}{l}\text { 1. Complement C4 precursor } \\
\text { 2. Chondroitin sulfate proteoglycan } 3 \\
\text { (neurocan) } \\
\text { 3. Hypothetical protein FLJ } 10298 \\
\text { 4. Heterogeneous nuclear } \\
\text { ribonucleoprotein M isoforms }\end{array}$ \\
\hline
\end{tabular}

ASAPRatio program. ${ }^{15}$ CSF proteins with Older versus Younger ASAP Ratio (OYR-ASAP) greater or less than $50 \%$ were agrin, chondroitin sulfate proteoglycan 3 , serine/threonine protein phosphatase PP-1 gamma catalytic subunit, and heterogeneous nuclear ribonucleoprotein M isoform (hnRNPm). We purchased all commercially available antibodies against the proteins (Table 2) that had OYR-ASAP that was increased or decreased $50 \%$. Western blot analysis was performed and relative levels of each protein quantified by measuring normalized optical densities of the corresponding bands. Only the antibodies to agrin and hnRNPm had sufficient sensitivity to detect protein in human CSF (data not shown). Consequently, we determined the levels of agrin and hnRNPm in individual CSF samples in a separate set of younger and older subjects (10 for each group). The results of Western blot analysis demonstrated that both proteins could differentiate older from younger subjects with about $30 \%$ sensitivity at greater than $95 \%$ specificity. However, combination of these two markers increased the sensitivity to $60 \%$ while maintaining $95 \%$ specificity, indicating that these two proteins likely are independent markers for aging.

Our next ICAT LC-ESI-MS-MS experiment compared pooled CSF from AD patients $(n=32)$ with agematched controls $(n=31)$. In combination with our previous study we identified a grand total of 390 proteins in human CSF. While most CSF proteins had an AD:C ASAP ratio (ACR-ASAP) between 0.9 and 1.1, 39 CSF proteins had ACR-ASAP $\geq 1.2$, while 46 had ACRASAP $\leq 0.8$ (Table 3). Interestingly, only 2 of 13 CSF proteins that showed $\geq 20 \%$ age-related change also showed $\geq 20 \%$ AD-related change, suggesting that processes of normal aging and $\mathrm{AD}$ are largely distinct. We pursued validation of our proteomic results by determining relative CSF levels of selected proteins in individual CSF samples by Western blot. Since the volume of remaining CSF was limiting, we selected those two proteins that that gave the most consistent results by both proteomic and pooled CSF Western blots; these were APP and cathepsin B precursor (CBP). Relative levels of both APP and CBP were significantly increased in CSF

TABLE 3. CSF Proteins Increased in AD versus Controls as Determined with ICAT LC-ESI-MS-MS and ASAPRatio ( \pm SEM)

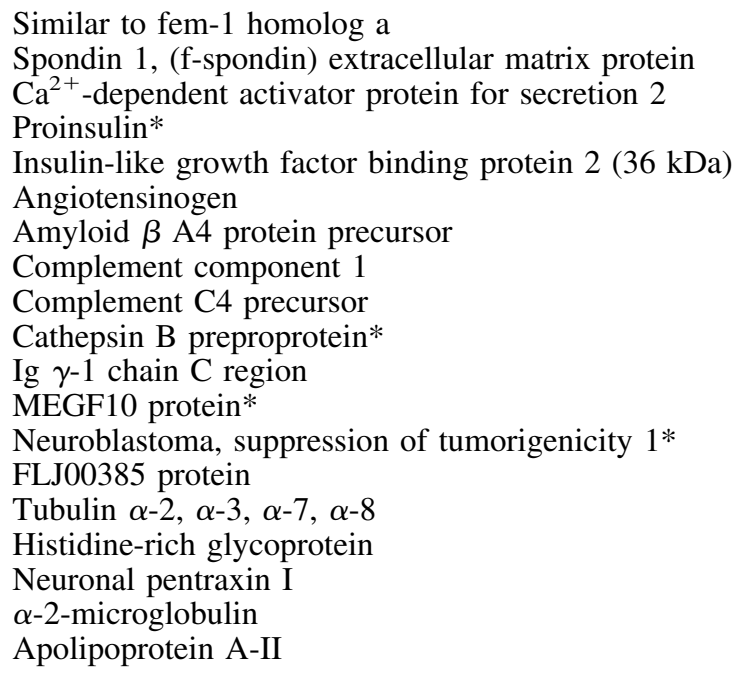

$$
\begin{array}{r}
11.1 \pm 0.1 \\
5.3 \pm 0.2 \\
3.9 \pm 0.3 \\
2.4 \pm 0.2 \\
2.3 \pm 1.2 \\
2.3 \pm 0.1 \\
2.2 \pm 1.0 \\
2.0 \pm 0.0 \\
1.9 \pm 0.1 \\
1.9 \pm 0.2 \\
1.8 \pm 0.8 \\
1.7 \pm 0.2 \\
1.6 \pm 0.6 \\
1.6 \pm 0.4 \\
1.5 \pm 0.2 \\
1.5 \pm 0.2 \\
1.5 \pm 0.2 \\
1.5 \pm 0.1 \\
1.5 \pm 0.1
\end{array}
$$

\footnotetext{
*Proteins identified by a single unique peptide.
} 
from AD patients compared with controls ( $p<0.02$ for both). The calculated average ( \pm SEM) AD:C ratios from these individual samples were $2.9 \pm 0.7$ and $1.3 \pm$ 0.1 for APP and CBP, respectively, values in good agreement with the ACR-ASAP from pooled CSF. The data for APP, the more discriminating single protein of the two validated by Western blot, had sensitivity of $67 \%$ and specificity of $90 \%$ for $\mathrm{AD}$ with a cutoff value of 0.07 relative optical density units. Adding CBP results to this analysis did not significantly alter sensitivity and specificity values.

\section{SUMMARY}

Systems biology offers enormous potential to understand the complexity of human brain aging and neurodegenerative diseases. Proteomics has an important role in these investigations because of its unique strengths and because of the potential central pathogenic contribution of pathological protein to several of these diseases. Here we have reviewed the methods and presented some examples of LC-ESI-MS-MS-based proteomics, with and without ICAT quantification, in the investigation of aging and AD. As protocols and methods for better quantitative high-throughput proteomics continue to improve, this approach will likely continue to provide deeper insight into human brain aging and neurodegenerative diseases. It is likely that this more comprehensive knowledge of the molecular underpinnings of aging and dementia will highlight new pathogenic mechanisms and therapeutic targets.

Acknowledgments: This work was supported by the Nancy and Buster Alvord Endowment, the Cheng-Mei Shaw Endowment, and Grants AG23801 and AG05136 from the National Institutes of Health. We deeply appreciate those who have donated tissue or CSF for our study.

\section{REFERENCES}

1. Wilson K, Ryan M, Prime J, Pashby D, Orange P, O'Beirne G, Whateley J, Bahn S, Morris C. Functional genomics and proteomics: application in neurosciences. J Neurol Neurosurg Psychiatry 75:529-538, 2004.

2. Ginsberg SD, Che S, Counts SE, Mufson EJ. Single cell gene expression profiling in Alzheimer's Disease. NeuroRx 3:302-317, 2006.

3. Miller PM, Federoff HJ. Microarrays in Parkinson's Disease: a systematic approach. NeuroRx 3:318-325, 2006.

4. Butterfield DA, Abdul HM, Newman S, Reed T. Redox proteomics in some age-related neurodegenerative disorders or models thereof. NeuroRx 3:343-356, 2006.

5. Choe LH, Werner BG, Lee KH. Two-dimensional protein electrophoresis: from molecular pathway discovery to biomarker discovery in neurological disorders. NeuroRx 3:326-334, 2006.

6. Lukiw WJ, Bazan NG. Cyclooxygenase 2 RNA message abundance, stability, and hypervariablity in sporadic Alzheimer's neocortex. J Neurosci Res 50:937-945, 1997.

7. Lahm HW, Langen H. Mass spectrometry: a tool for the identification of proteins separated by gels. Electrophoresis 21:21052114,2000
8. Lopez MF, Pluskal MG. Protein micro- and macroarrays: digitizing the proteome. J Chromatogr B Analyt Technol Biomed Life Sci 787:19-27, 2003.

9. Aebersold R, Goodlett D. Mass spectrometry in proteomics. Chem Rev 101:269-295, 2001.

10. Gygi SP, Rist B, Gerber SA, Turecek F, Gelb MH, Aebersold R. Quantitative analysis of complex protein mixtures using isotopecoded affinity tags. Nat Biotechnol 17:994-999, 1999.

11. Goodlett DR, Yi EC. Proteomics without polyacrylamide: qualitative and quantitative uses of tandem mass spectrometry in proteome analysis. Funct Integr Genomics 2:138-153, 2002.

12. Foret F, Preisler J. Liquid phase interfacing and miniaturization in matrix-assisted laser desorption/ionization mass spectrometry. Proteomics 2:360-372, 2002.

13. Morris HR, Paxton T, Dell A, Langhorne J, Berg M, Bordoli RS, Hoyes J, Bateman RH. High sensitivity collisionally-activated decomposition tandem mass spectrometry on a novel quadrupole/ orthogonal-acceleration time-of-flight mass spectrometer. Rapid Commun Mass Spectrom 10:889-896, 1996.

14. Wu SL, Jardine I, Hancock WS, Karger BL. A new and sensitive on-line liquid chromatography/mass spectrometric approach for top-down protein analysis: the comprehensive analysis of human growth hormone in an E. coli lysate using a hybrid linear ion trap/Fourier transform ion cyclotron resonance mass spectrometer. Rapid Commun Mass Spectrom 18:2201-2207, 2004.

15. Li XJ, Zhang H, Ranish JA, Aebersold R. Automated statistical analysis of protein abundance ratios from data generated by stableisotope dilution and tandem mass spectrometry. Anal Chem 75: 6648-6657, 2003.

16. Ross PL, Huang YN, Marchese JN, Williamson B, Parker K, Hattan S, Khainovski N, et al. Multiplexed protein quantitation in Saccharomyces cerevisiae using amine-reactive isobaric tagging reagents. Mol Cell Proteomics 3:1154-1169, 2004.

17. Hardy J, Selkoe DJ. The amyloid hypothesis of Alzheimer's disease: progress and problems on the road to therapeutics. Science 297:353-356, 2002.

18. Levy-Lahad E, Tsuang D, Bird TD. Recent advances in the genetics of Alzheimer's disease. J Geriatr Psychiatry Neurol 11:42-54, 1998.

19. Trojanowski JQ, Lee VM. Phosphorylation of paired helical filament tau in Alzheimer's disease neurofibrillary lesions: focusing on phosphatases. FASEB J 9:1570-1576, 1995.

20. Spillantini MG, Bird TD, Ghetti B. Frontotemporal dementia and Parkinsonism linked to chromosome 17: a new group of tauopathies. Brain Pathol 8:387-402, 1998.

21. Namba Y, Tomonaga M, Kawasaki H, Otomo E, Ikeda K. Apolipoprotein $\mathrm{E}$ immunoreactivity in cerebral amyloid deposits and neurofibrillary tangles in Alzheimer's disease and kuru plaque amyloid in Creutzfeldt-Jakob disease. Brain Res 541:163-166, 1991.

22. Lowe JS, Leigh N. Disorders of movement and system degenerations. In: Greenfield's neuropathology (Graham DI, Lantos PL, eds), Vol 2, pp 325-430. New York: Arnold Publishing, 2002.

23. Liao L, Cheng D, Wang J, Duong DM, Losik TG, Gearing M, et al. Proteomic characterization of postmortem amyloid plaques isolated by laser capture microdissection. J Biol Chem 279:3706137068, 2004.

24. Wang Q, Woltjer RL, Cimino PJ, Pan C, Montine KS, Zhang J, Montine TJ. Proteomic analysis of neurofibrillary tangles in Alzheimer disease identifies GAPDH as a detergent-insoluble paired helical filament tau binding protein. FASEB J 19:869-871, 2005.

25. Sirover MA. New insights into an old protein: the functional diversity of mammalian glyceraldehyde-3-phosphate dehydrogenase. Biochim Biophys Acta 1432:159-184, 1999.

26. Mazzola JL, Sirover MA. Alteration of intracellular structure and function of glyceraldehyde-3-phosphate dehydrogenase: a common phenotype of neurodegenerative disorders? Neurotoxicology 23:603-609, 2002.

27. Mazzola JL, Sirover MA. Subcellular alteration of glyceraldehyde3-phosphate dehydrogenase in Alzheimer's disease fibroblasts. J Neurosci Res 71:279-285, 2003.

28. Li Y, Nowotny P, Holmans P, Smemo S, Kauwe JS, Hinrichs AL, et al. Association of late-onset Alzheimer's disease with genetic 
variation in multiple members of the GAPD gene family. Proc Natl Acad Sci USA 101:15688-15693, 2004.

29. Montine TJ, Shinobu L, Montine KS, Roberts II LJ, Beal MF, Morrow JD. No difference in plasma or urine $F_{2}$-isoprostanes among patients with Huntington's disease or Alzheimer's disease, and controls. Ann Neurol 48:950, 2000.

30. Montine TJ, Quinn JF, Milatovic D, Silbert LC, Dang T, Sanchez $\mathrm{S}$, et al. Peripheral $\mathrm{F}_{2}$-isoprostanes and $\mathrm{F}_{4}$-neuroprostanes are not increased in Alzheimer's disease. Ann Neurol 52:175-179, 2002.

31. Andreasen N, Sjogren M, Blennow K. CSF markers for Alzheimer's disease: total tau, phospho-tau and A $\beta 42$. World J Biol Psychiatry 4:147-155, 2003.

32. Clark C, Xie S, Chittams J, Ewbank D, Peskind E, Galasko D, et al. Cerebrospinal fluid tau and beta-amyloid: how well do these biomarkers reflect autopsy-confirmed dementia diagnoses? Arch Neurol 60:1696-1702, 2003.

33. Galasko D. Cerebrospinal fluid biomarkers in Alzheimer's disease: a fractional improvement? Arch Neurol 60:1195-1196, 2003.

34. Montine TJ, Neely MD, Quinn JF, Beal MF, Markesbery WR, Roberts LJ II, Morrow JD. Lipid peroxidation in aging brain and Alzheimer's disease. Free Radic Biol Med 33:620-626, 2002.

35. Roberts LJ II, Montine TJ, Markesbery WR, Tapper AR, Hardy P, Chemtob S, et al. Formation of isoprostane-like compounds (neuroprostanes) in vivo from docosahexaenoic acid. J Biol Chem 273:13605-13612, 1998.

36. Zhang J, Goodlett DR, Peskind ER, Quinn JF, Zhou Y, Wang Q, et al. Quantitative proteomic analysis of age-related changes in human cerebrospinal fluid. Neurobiol Aging 26:207-227, 2005.

\section{Receive tables of contents by e-mail}

To receive tables of contents by e-mail, sign up through our website at

www.neurorx.org.

\section{Instructions}

Log on to www.neurorx.org and click "Email alert" in the Features section of the home page. Complete the registration process. You will receive an e-mail message confirming that you have been added to the mailing list. Note that tables of contents emails will be sent when a new issue is posted to the Web. 